\title{
The SuperB Project
}

\section{G. Finocchiaro*†}

INFN - Laboratori Nazionali di Frascati

E-mail: giuseppe.finocchiaroelnf.infn.it

The SuperB project for a next generation asymmetric $e^{+} e^{-}$flavor factory to be built in the Rome area with a baseline luminosity of $10^{36} \mathrm{~cm}^{-2} \mathrm{~s}^{-1}$ is discussed. Some explicit examples are given to elucidate how such a facility can provide a uniquely sensitive probe of physics beyond the Standard Model. The basic accelerator concepts allowing luminosities 50-100 times larger than the existing $B$ factories are briefly discussed, along with the main characteristics of the Super $B$ detector.

The Xth Nicola Cabibbo International Conference on Heavy Quarks and Leptons,

October 11-15, 2010

Frascati (Rome) Italy

\footnotetext{
*Speaker.

$\dagger$ On behalf of the SuperB Collaboration
} 


\section{Introduction}

New physics (NP) is generally expected beyond the Standard Model of High Energy Physics (SM), although the energy scale $\Lambda$ of the supposed new interactions is not univocally predicted. The theoretically well-motivated possibility that $\Lambda$ is around $1 \mathrm{TeV}$ could make the NP accessible to the Large Hadron Collider (LHC). In such a case SuperB could study the flavor structure of NP, measure the flavor couplings and search for still heavier mass states. Alternatively, the NP energy scale could lie above the direct reach of LHC. In such a scenario SuperB can look for indirect NP signals, understand where they may come from, and exclude regions in the multi-dimensional parameter space of NP models up to $\Lambda \sim 10 \mathrm{TeV}$ or more. In addition to probing energy scales higher than $\mathrm{LHC}$ via virtual processes, $\operatorname{Super} B$ can, thanks to its clean $e^{+} e^{-}$environment, experimentally access several physics channels precluded to hadronic machines as LHC. Those channels include decays with neutrinos or neutral particles in the final state, or whenever an inclusive analysis is required, as for example $B F\left(B \rightarrow K^{(*)} v \bar{v}\right), B F\left(B \rightarrow X_{s} \gamma\right)$, and the measurement of the $\left|V_{u b}\right|$ or $\left|V_{c b}\right|$ CKM matrix elements.

In order to achieve the sensitivity goals of the project (some relevant examples will be discussed in the next section), a dataset of about $75 \mathrm{ab}^{-1}\left(80 \times 10^{9} \mathrm{~B} \bar{B}\right.$ pairs) is required, which could be collected at the $\Upsilon(4 S)$ in 5 years of data taking if $\mathscr{L}=10^{36} \mathrm{~cm}^{-2} \mathrm{~s}^{-1}$, assuming that the accelerator and detector efficiency are kept at the very high levels of the $B$ factories. It is important to remark that Super $B$ will also produce large samples of $D$ meson $\left(100 \times 10^{9}\right)$ and $\tau$ lepton $\left(70 \times 10^{9}\right)$ pairs, allowing for NP searches in the up-type quark and lepton sectors with unprecedented precision. The ability of SuperB to vary the center-of-mass energy from the charm and $\tau$ production threshold up to the $\Upsilon(5 S)$, and to have at least one beam with $80 \%$ longitudinal polarization, will further boost the physics potential of the experiment.

As demonstrated by the several hundred papers published by the $B$-factory experiments, a wide range of important measurements can be performed in the clean $e^{+} e^{-}$environment at $\Upsilon(4 S)$. Most of these are statistics-limited, and would therefore improve substantially with a data sample of $75 \mathrm{ab}^{-1}$. In many cases, large control samples can be used to further reduce systematic and theoretical errors. Control of the theoretical errors (e.g. those related to lattice calculations) is particularly relevant to fully exploit the statistical power of the experimental measurements. New developments in unquenched lattice calculation techniques, together with the expected increase in computing power, will allow, by the time the data will be collected, to match the Super $B$ experimental precision [1]. With $75 \mathrm{ab}^{-1}$ Super $B$ will be able to substantially improve on the precision of the CKM Unitarity Triangle (UT) parameters. For example, the present error on the $\bar{\rho}$ and $\bar{\eta}$ parameters of the UT ( \pm 0.028 and \pm 0.016 respectively) could be reduced [2] to $\delta \bar{\rho}= \pm 0.0028$, $\delta \bar{\eta}= \pm 0.0024$. The possibility to study a very large numbers of physical observables, and the correlations among them, is a particularly important tool to elucidate the nature of new physics, should deviations with respect to SM predictions be observed.

\section{SuperB Physics Highlights}

In this section a few examples of physics channels in which the Super $B$ can give significant contributions are briefly reviewed. For an extensive discussion, we refer the reader to [1]. 


\section{$2.1 B \rightarrow K^{(*)} v \bar{v}$}

The rare $B \rightarrow K^{(*)} v \bar{v}$ decays are interesting probes of new physics from $Z^{0}$ penguins [3]. Moreover, since the neutrinos escape the detector unmeasured, the $b \rightarrow s+E_{\text {miss }}$ channel could be affected by exotic sources of missing energy, such as e.g. light dark matter [4]. The $B \rightarrow K v \bar{v}$ branching fraction, with an experimental upper bound only a factor of three above the SM prediction, currently provides the most stringent constraint on NP. In the $B \rightarrow K^{*} v \bar{v}$ decay one can also measure the longitudinal polarization fraction $f_{L}\left(q^{2}\right)$, which is theoretically very clean since form factor uncertainties cancel to a large extent [5]. A detailed analysis based on the recoil technique was performed to extrapolate the Super $B$ reach on the $B \rightarrow K^{(*)} v \bar{v}$ decays from the BABAR measurement. In the recoil technique one $B$ meson is fully reconstructed, yielding a high purity sample with known kinematics, flavor and charge. The improved Super $B$ hermeticity (see later) is crucial in background-dominated, very rare channels. In Super $B$ an increase of about $25 \%$ with respect to $B A B A R$ in the $\mathrm{S} / \mathrm{B}$ ratio is expected.

In a general NP model $B F(B \rightarrow K v \bar{v}), B F\left(B \rightarrow K^{*} v \bar{v}\right), f_{L}\left(q^{2}\right)$ and the inclusive branching fraction $B F\left(B \rightarrow X_{s} v \bar{v}\right)$ can be expressed in terms of the two real parameters $\varepsilon$ and $\eta$, which can be over-constrained by the four measurements ( $\varepsilon=1, \eta=0$ in the SM). Fig. 1 shows the correlation among the observables in the $(\varepsilon, \eta)$ plane (left), and how the constraint in the plane could be improved at $\operatorname{Super} B$ (right).
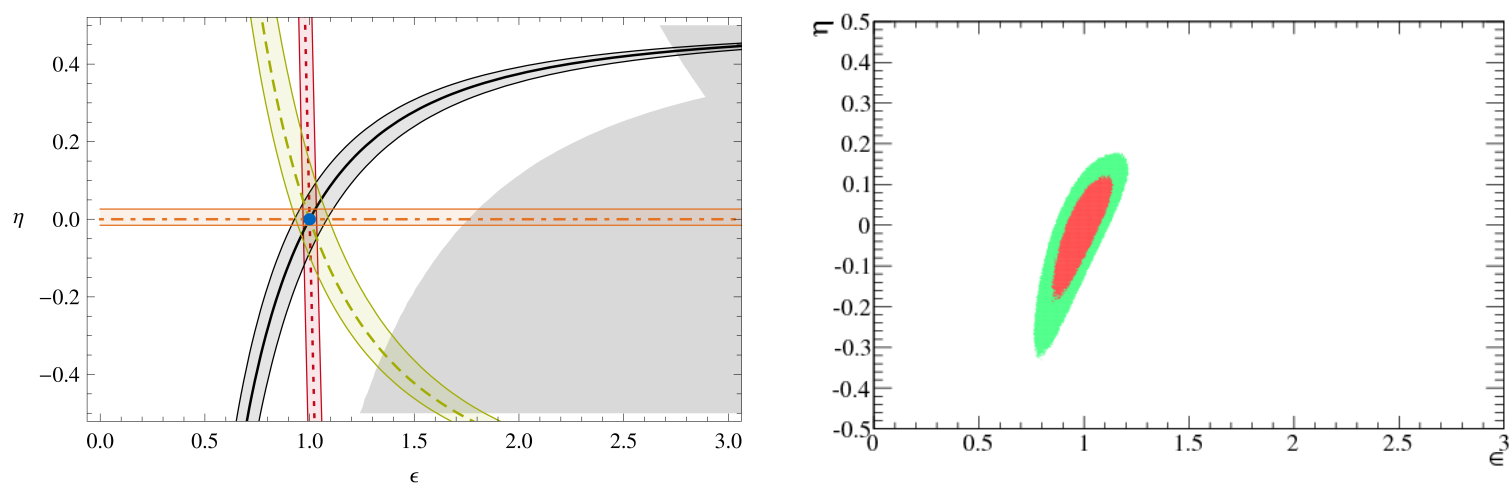

Figure 1: Left: constraints on the $\varepsilon-\eta$ plane, where the bands for $B F(B \rightarrow K v \bar{v})$ (green), $B F\left(B \rightarrow K^{*} v \bar{v}\right)$ (black), $f_{L}\left(q^{2}\right)$ (orange) and $B F\left(B \rightarrow X_{S} v \bar{v}\right)$ (red) only account for theoretical errors [5]. The grey area is excluded at $90 \%$ CL by present data. Right: expected constraint on the $(\varepsilon, \eta)$ plane, from the measurement of the branching fractions of $B \rightarrow K^{(*)} v \bar{v}$ decays and the angular analysis of $B^{0} \rightarrow K^{* 0} v \bar{v}$ with $75 \mathrm{ab}^{-1}$.

\subsection{LFV in $\tau$ Decays}

Lepton flavor violation (LVF) in $\tau$ decays, which is negligibly small in the SM but is enhanced in several SM extensions, is another very sensitive NP probe. The search for LFV has already been actively pursued at the $B$ factories, which pushed the $90 \%$ CL branching fraction limits in almost 50 different decay modes down to a few $10^{-8}$. From an extrapolation of the BABAR analysis, the Super $B$ sensitivities in the "golden" LFV channels $\tau \rightarrow \mu \gamma$ and $\tau \rightarrow \mu \mu \mu \gamma$ are $2.4 \times 10^{-9}$ and $2.3 \times 10^{-9}$ respectively. The ratio of branching fractions to these modes could distinguish between 
the SUSY and Little Higgs models [2]. In addition to this, the limit on $\tau \rightarrow \mu \gamma$, when combined with constraints on $\mu \rightarrow e \gamma$ from MEG and $\vartheta_{13}$ from accelerator and reactor neutrino experiments, can be used to distinguish among different NP scenarios [2].

As a key feature of its baseline design, SuperB incorporates an $80 \%$ polarized electron beam (polarization of the $e^{+}$beam is more difficult to realize and will be considered as an upgrade). The angular distribution of the decay products of polarized $\tau$ 's depends on the particular decay, and can be used to effectively suppress backgrounds (a typical example being $\tau \rightarrow \mu \nu \bar{v} \gamma$ for the LVF decay $\tau \rightarrow \mu \gamma$ ). The polarization is also instrumental in improving the sensitivity on the $\tau$ EDM and $g-2$.

\subsection{Charm at Threshold}

The measurement of $D^{0} \bar{D}^{0}$ oscillations has opened a new window to search for $C P$ violation in charm which, if observed, would provide unequivocal NP signals. With $75 \mathrm{ab}^{-1}$ at $\Upsilon(4 S)$ Super $B$ will dramatically improve (by a factor 12) the precision in the determination of the $D^{0} \bar{D}^{0}$ mixing parameters. The strong phase difference $\delta_{f}$ between the produced $D^{0}$ and $\bar{D}^{0}$ cannot however be measured at $\Upsilon(4 S)$, but only at the charm production threshold, where the $D \bar{D}$ pairs exhibit quantum coherence. With $0.5 \mathrm{ab}^{-1}$ at the $\psi(3770)$ Super $B$ can measure $\delta_{f}$ to $\pm 1^{\circ}$, several flavorchanging neural current modes with sensitivities of the order of $10^{-8}$, and finally strongly reduce the Dalitz-plot model uncertainty in the $\gamma$ angle measurement.

\section{SuperB Accelerator Highlights}

The Super $B$ collider exploits a novel collision scheme [6,7], based on very small beam dimensions and betatron function at the interaction point, on large crossing and Piwinsky angle ${ }^{1}$ and on the "crab waist" scheme. This approach allows to reach the required luminosity of $10^{36} \mathrm{~cm}^{-2} \mathrm{~s}^{-1}$ and at the same time overcome the difficulties of early super $e^{+} e^{-}$collider designs, most notably very high beam currents and very short bunch lengths. The wall-plug power and the beam-related background rates in the detector are therefore kept within affordable levels [8].

The crab waist transformation consists in moving the waist of each beam onto the axis of the other beam with a pair of sextupole up- and down-stream the IP. In this way all particles from both beams collide in the minimum $\beta_{y}^{*}$ region, with a net luminosity gain. Moreover (and most significantly) the $x / y$ betatron resonances are naturally suppressed. The principle of the innovative IR design sketched above has been experimentally demonstrated at the Frascati Da $\phi$ NE collider [9]. Very importantly, this test also validated the simulations used to calculate the IR optics.

Several other accelerator-physics ideas critical to the success of the SuperB project have also been realized in practice, either in past colliders or as part of accelerator R\&D activities such as those for the Linear Collider: KEK-B and PEP-II have worked very well with asymmetric interaction regions, storing 2-3A of beam currents and performing continuous injection with live detectors; polarized beams have been successfully produced at the SLC, and spin manipulation tests have been performed at Novosibirsk; finally, ultra-low emittance lattices were tested for the ILC damping rings. This long list of achievements, and the fact that the luminosity of $10^{36} \mathrm{~cm}^{-2} \mathrm{~s}^{-1}$ is

\footnotetext{
${ }^{1}$ The luminosity formula for $e^{+} e^{-}$beams colliding with an horizontal crossing angle $\vartheta$ can be expressed [7] in terms of the vertical tune shift parameter $\xi_{y}$, the vertical beta function at the IP $\beta_{y}$ and the number of particles per bunch $N$ (proportional to the beam current) as $\mathscr{L} \propto N \xi_{y} / \beta_{y}$, with $\xi_{y} \propto N \beta_{y} /\left(\sigma_{x} \sigma_{y} \sqrt{1+\phi^{2}}\right)$. The Piwinsky angle $\phi \simeq \vartheta \sigma_{x} / \sigma_{y}$.
} 
not a singularity in the parameter space but can be obtained with different settings of the accelerator parameters, as $e . g$. the $x$ and $y$ emittances and $\beta_{y}^{*}$ in both rings, the vertical tune shift, the beam currents, adds confidence that this unprecedented luminosity can indeed be obtained in practice.

The Super $B$ design is based on recycling as much as possible existing PEP-II hardware, with a significant reduction of costs. The optimal beam energy choice for accelerator design, including polarization, is $4.18 \mathrm{GeV}$ positron beam on $6.71 \mathrm{GeV}$ electron beam.

The low currents, ultra-small emittance approach has been adopted recently also by the KEKB accelerator team, which defined a new set of parameters very similar to that of the Italian Super $B$.

\section{SuperB Detector Highlights}

Most of the general requirements for the Super $B$ detector are common to those of the present $B$ factories, including large solid angle coverage, good particle identification (PID) capabilities over a wide momentum range $(\pi / K$ separation to over $4 \mathrm{GeV} / c)$, measurement of the relative decay times of the $B$ mesons, good resolution on the momentum of charged tracks and on the photon energy, particularly in the sub- $\mathrm{GeV}$ part of the spectrum, relevant at the $\Upsilon(4 S)$ environment. The Super $B$ detector concept is therefore based on the BABAR detector, with the modifications required to operate at a much higher luminosity (and luminosity-scaling background rates), and with a reduced center-of-mass boost [10].

The BABAR detector is composed by a tracking system - a five layer double-sided silicon strip vertex tracker (SVT) and a 40 layer drift chamber (DCH) immersed in a $1.5 \mathrm{~T}$ magnetic field - a Cherenkov detector with fused silica bar radiators (DIRC), a homogeneous electromagnetic calorimeter made of $\mathrm{CsI}(\mathrm{Tl})$ crystals (EMC), and a detector for muon identification and $K_{L}^{0}$ detection (IFR) realized instrumenting the iron flux return with resistive plate chambers and limited streamer tubes. Super $B$ is designed to reuse a number of BABAR components: the DIRC quartz bars, the $\mathrm{CsI}(\mathrm{Tl})$ crystals of the barrel EMC, the flux-return steel, the superconducting coil.

The center-of-mass boost at $\operatorname{Super} B$ is smaller than in $B A B A R$ ( $\beta \gamma=0.24$ vs. 0.56). While this effectively improves the angular coverage of the detector, it also reduces the $\Delta z$ separation of the decay vertices. The $\Delta t$ sensitivity in time-dependent measurements is maintained by improving the vertex resolution: the Super $B$ vertex detector replicates the five-layer BABAR SVT, but exploits the reduced dimensions of the beam pipe made possible by the ultra-low emittance SuperB beams to add a very thin and precise measurement layer at a radius of only $1.5 \mathrm{~cm}$. The baseline technology for this "Layer0" uses short double-sided silicon strip detectors ("striplets"), while other options are being considered as possible upgrades. The SuperB DCH concept is derived from the BABAR one, with several improvements: the mechanical structure entirely in Carbon-Fiber composite, resulting in a 4-fold reduction of the endplate material; the gas mixture and the wire cell layout optimized to minimize the multiple-scattering contribution to momentum resolution; the readout electronics redesigned to cope with the higher trigger rate, and minimize the FEE material. The hadron PID system will use the radiator quartz bars of the BABAR DIRC, read-out by fast multi-anode PMTs, and with the imaging region considerably reduced in size to improve performance and reduce the impact of backgrounds. The forward EMC will feature cerium-doped LYSO crystals, which have a much shorter scintillation time constant, a smaller Molière radius and better radiation hardness than the current $\mathrm{CsI}(\mathrm{Tl})$ crystals, for reduced sensitivity to beam backgrounds and better position 
resolution. The thickness of the flux-return iron will be increased with additional absorber to bring to about 7 the number of interaction lengths for muons, while the gas detectors will be replaced by extruded plastic scintillator bars to cope with the expected background rates. Finally, the Collaboration is considering to improve the detector hermeticity by inserting a "veto-quality" lead-scintillator EMC calorimeter in the backward direction, and to add a particle identification device in front of the forward calorimeter. Both detectors would improve the sensitivity to rare modes such as $B \rightarrow K^{(*)} v \bar{v}$ and $B \rightarrow \tau v$. A concept of the Super $B$ detector is shown in Fig. 2.

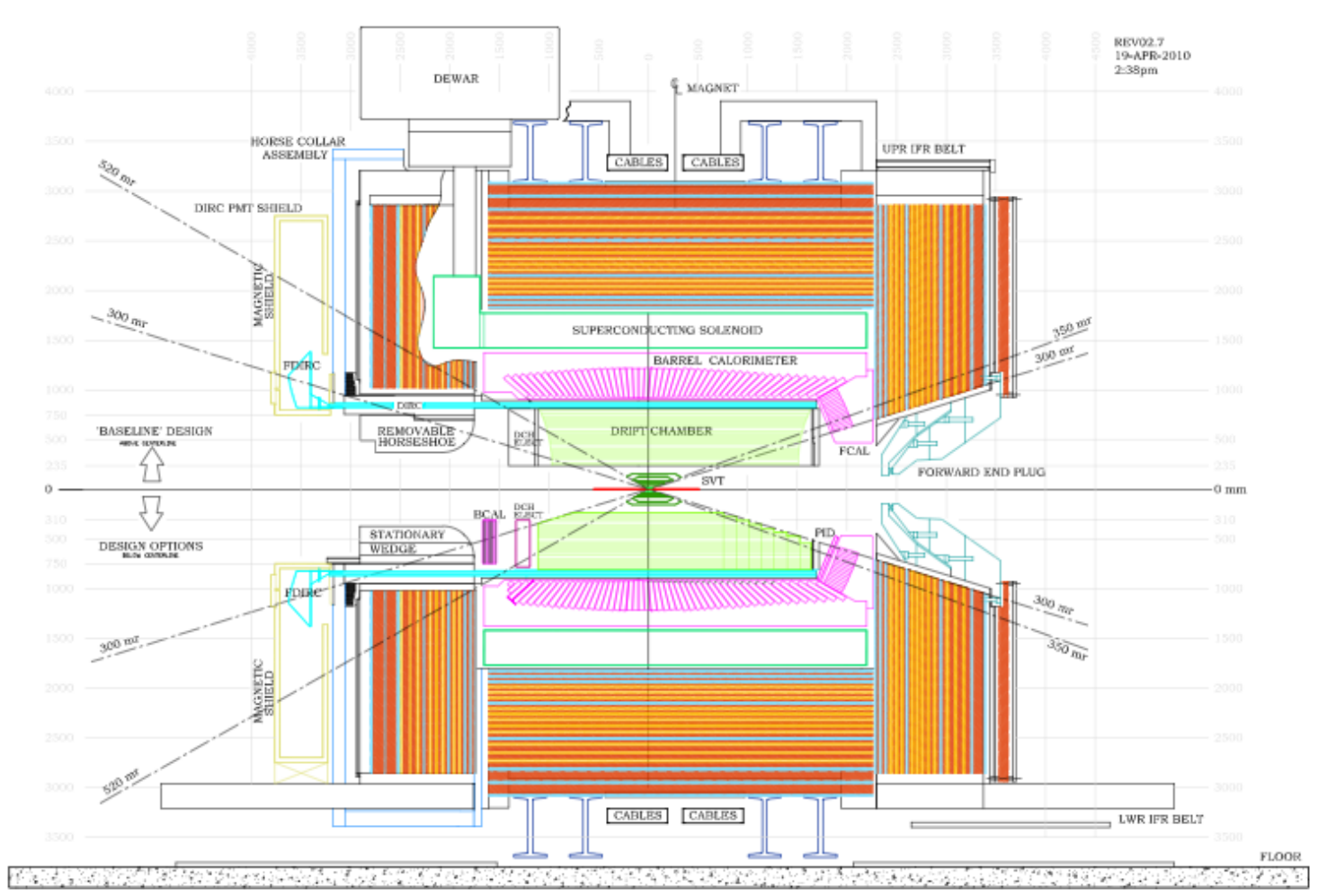

Figure 2: Concept for the SuperB detector. The upper half shows the baseline concept, and the bottom half adds a number of optional detector configurations.

\section{Summary}

We concisely presented the Super $B$ physics program, discussing some examples of key measurements possible at $\Upsilon(4 S)$ and $\psi(3770)$ center-of-mass energies, and the importance of incorporating longitudinal beam polarization in the design. The main features of the accelerator and detector projects were briefly described. An exhaustive discussion of all SuperB aspects can be found in $[1,8,10]$.

\section{References}

[1] B. O'Leary et al.: SuperB Progress Report - Physics; arXiv:1008.1541v1 [hep-ex]. 
[2] M. Bona et al.: SuperB: A High-Luminosity Asymmetric $e^{+} e^{-}$Super Flavor Factory. Conceptual Design Report. SLAC-R-856, INFN-AE-07-02, LAL-07-15 (2007), 480pp (arXiv:0709.0451 [hep-ex]).

[3] G. Buchalla, G. Hiller and G. Isidori: Phys. Rev. D 63 (2001) 014015 [arXiv:hep-ph/0006136].

[4] C. Bird, P. Jackson, R. Kowalewski, M. Pospelov: Phys.Rev.Lett.93:201803,2004.

[5] W. Altmannshofer, A. J. Buras, D. M. Straub and M. Wick: JHEP 0904 (2009) 022.

[6] P. Raimondi: Talk given at the $2^{\text {nd }}$ Super B-Factory Workshop, http://www. Inf.infn.it/conference/superb06/talks/raimondil.ppt

[7] P. Raimondi, D. Shatilov, M. Zobov: arXiv:physics/0702033v1 [physics.acc-ph].

[8] M. E. Biagini et al.: SuperB Progress Report - Accelerator; arXiv:1009.6178 [physics.acc-ph].

[9] The DAФNE Team: Phys. Rev. Lett. 104, 174801 (2010).

[10] E. Grauges et al.: SuperB Progress Report - Detector; arXiv:1007.4241v1 [hep-ex]. 